\title{
Composição mineralógica e geoquímica dos solos do parque estadual do Itacolomi - Ouro Preto/MG
}

\section{Mineralogical composition and heavy metal contents of soils from the Itacolomi state park - Ouro Preto/MG}

\author{
Larice Nogueira Andrade $^{\text {ac }}$, Mariangela Garcia Praça Leite ${ }^{\text {bd }}$, Luís de Almeida Prado \\ Bacellar ${ }^{\text {be }}$ \\ ${ }^{\mathbf{a}}$ Universidade Federal do Espírito Santo, ${ }^{\mathbf{b}}$ Universidade Federal de Ouro Preto

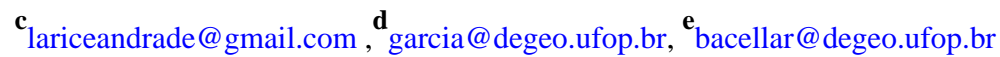

\begin{abstract}
RESUMO
O objetivo deste trabalho foi estudar a mineralogia e geoquímica dos solos do Parque Estadual do Itacolomi, Ouro Preto (MG), e relacioná-las aos cenários geológicos da região. Foram selecionados seis locais, de onde se coletaram amostras em seis profundidades $(0-10,10-20$, 20-30, 30-40, 40-50 e 50-60 cm), representando áreas das diferentes unidades litológicas da região. Os elementos químicos foram determinados por meio do ICP-OES, e a determinação mineralógica dos solos foi realizada por difração de raios-X. Os resultados das análises demonstraram que a geologia exerce grande influência nas características do solo. Os elementos químicos obtidos nos solos analisados foram comparados com valores-padrões definidos pela norma CETESB (2005), possibilitando a comprovação da influência geológica na qualidade dos solos, onde foram detectadas concentrações anômalas de diversos elementos. Pelo fato de a área de estudo ser uma Unidade de Conservação, com menor possibilidade de interferência antrópica, tais anomalias representam concentrações naturais, ou seja, são reflexo da litologia.
\end{abstract}

Palavras-chave: Contaminação do solo; Teores naturais; Metais em solos.

\begin{abstract}
The purpose of this study was to investigate the mineralogy and geochemistry contents of soils from the Itacolomi State Park, in Ouro Preto (MG), and their relationship to the geological scenario. The samples were collected at six depths $(0-10,10-20,20-30,30-40,40-50$ e $50-60$ $\mathrm{cm})$ in representative areas from the different lithologies. The chemical elements were determined by ICP-OES and the soil mineralogy determination was performed by X-rays diffraction. The results of the analisys show the geology influence under the soil features. The chemical elements from the soils were compared with standard values defined by the rule CETESB (2005) proving the geological influences on the soil quality. Outliers for several elements were detected in soils. These outliers can be related the lithology indicating natural concentrations because the explored area is a preservation unit without anthropogenic interference.
\end{abstract}

Keywords: Soil contamination; Baselines levels; Metals in soil.

\section{Introdução}

O controle da poluição das águas, do ar e dos solos revela-se como um dos maiores desafios da sociedade moderna. A intensificação das atividades industriais, agrícolas e urbanas tem causado considerável impacto ambiental nesses recursos. Com o intuito de avaliar e controlar esses impactos surgem importantes instrumentos legais (como as Resoluções CONAMA).
Tais resoluções tratam, sobretudo, da qualidade do ar e da água. Apesar das resoluções existirem desde a década de 1980, até recentemente não existia uma específica para a qualidade do solo. Apenas o estado de São Paulo dispunha de uma normativa com parâmetros e valores indicadores para a qualidade do solo (CETESB 2005). Por isso, na tentativa de reverter esse fato, o Ministério do Meio Ambiente promulgou recentemente a Resolução 420/2009 (CONAMA 
2009), que dispõe sobre critérios e valores orientadores de qualidade do solo e estabelece diretrizes para o gerenciamento ambiental de áreas contaminadas.

Para a implantação da Resolução 420/2009, os órgãos ambientais competentes dos estados e do Distrito Federal deverão levantar os tipos de solo em seus territórios e definir os Valores de Referência de Qualidade (VRQ), que são essenciais para a definição de áreas contaminadas.

Os VRQ ou de background são as concentrações naturais de determinado elemento em solos sem influência antrópica (Gough et al. 1994). Os principais contaminantes, de natureza inorgânica, que ocorrem naturalmente nos solos são os metais pesados. Metais pesados são elementos químicos que possuem densidade maior que $6 \mathrm{~g} / \mathrm{cm}^{3}$ (Alloway 1993). É uma denominação largamente aplicada para elementos frequentemente associados a problemas ambientais. Dessa forma, o termo metal pesado é também comumente aplicado para alguns elementos, que embora possuam densidades menores que $6 \mathrm{~g} / \mathrm{cm}^{3}$, apresentam efeitos tóxicos ao meio ambiente. Os principais elementos considerados tóxicos ao meio ambiente são o arsênio (As), cádmio (Cd), cromo (Cr), cobalto $(\mathrm{Co})$, chumbo $(\mathrm{Pb})$, cobre $(\mathrm{Cu})$, mercúrio $(\mathrm{Hg})$, níquel $(\mathrm{Ni})$, zinco $(\mathrm{Zn})$, ferro $(\mathrm{Fe})$, manganês $(\mathrm{Mn})$ e alumínio (Al).

A ocorrência natural de metais pesados em solos é fortemente influenciada pelo material de origem, ou seja, pela litologia da região (Oliveira 1996, Tiller 1989, Mitchell 1964). Além da natureza geológica, outras características do solo podem influenciar no conteúdo de metais pesados, tais como a composição mineralógica, os teores de matéria orgânica e as características físico-químicas dos solos (Klamt \& Van Reeuwijk 2000, Souza et al. 1996, Barona \& Romero 1996). Fadigas et al. (2006) propuseram o estabelecimento de valores de Background de Cd, Co, $\mathrm{Cr}, \mathrm{Cu}, \mathrm{Ni}, \mathrm{Pb}$ e $\mathrm{Zn}$ em um solo, a partir dos teores de silte, argila, manganês e ferro e da capacidade de troca catiônica (CTC).

De acordo com Paye (2008), o conhecimento das concentrações naturais de um determinado elemento é o mais simples e direto método para a definição de valores de referência. Burak (2008) destaca que a melhor forma de se identificar áreas poluídas ou contaminadas em um solo, por ação antrópica, é a sua comparação com um solo no mesmo contexto pedológico, porém sob condições naturais.

No Brasil, os valores médios de concentração de metais pesados são bastante heterogêneos, devido a sua grande variabilidade geológica e pedológica. Por isso, é importante que a adoção dos valores de referência de qualidade seja adequada às especificações locais. Hursthouse (2001) ressalta que o conhecimento dos valores de background local poderá evitar subestimar ou superestimar os níveis de contaminação.

\section{Material e métodos}

\subsection{Descrição da área e dos procedimentos de coleta}

O Parque Estadual do Itacolomi (PEI) localiza-se nos municípios de Ouro Preto e Mariana, na região do Quadrilátero Ferrífero do estado de Minas Gerais.

O relevo da área do PEI pode ser separado, pelo menos, em três unidades, que muitas vezes interagem entre si: vertente de topo convexo, regiões planas e relevo escarpado com cristas isoladas. Segundo Castañeda (1993), ocorrem dois tipos básicos de solo: os litossolos e os latossolos, que refletem as características do material de origem (litologia) e relevo (geoforma).

Tabela 1: Coordenadas geográficas dos pontos amostrados

\begin{tabular}{|c|c|c|}
\hline \multirow{2}{*}{$\begin{array}{c}\text { Pontos } \\
\text { amostrados }\end{array}$} & \multicolumn{2}{|c|}{ Coordenadas geográficas } \\
\hline & Latitude (S) & Longitude (W) \\
\hline Man & $20^{0} 25^{\prime} 98^{\prime \prime}$ & $43^{0} 30^{\prime} 59^{\prime \prime}$ \\
\hline Cal & $20^{0} 25^{\prime} 61^{\prime}$ & $43^{0} 29^{\prime} 86^{\prime \prime}$ \\
\hline Ben & $20^{0} 25^{\prime} 39^{\prime \prime}$ & $43^{0} 25^{\prime} 26^{\prime \prime}$ \\
\hline $\mathrm{Cd}$ & $20^{0} 27^{\prime} 24^{\prime}$ & $43^{0} 29^{\prime} 08^{\prime \prime}$ \\
\hline Md & $20^{0} 27^{\prime} 36^{\prime \prime}$ & $43^{0} 26^{\prime} 06^{\prime \prime}$ \\
\hline $\mathrm{Br}$ & $20^{0} 26^{\prime} 41^{\prime \prime}$ & $43^{0} 25^{\prime} 13^{\prime \prime}$ \\
\hline
\end{tabular}

No compartimento de cristas isoladas e relevo escarpado, predominam os litossolos. Associados aos quartzitos, apresentam textura arenosa, são essencialmente rasos e bastante limitados em termos de fertilidade (Ferreira \& Lazarin 1993). Os latossolos são encontrados na área de vertentes de topos convexos e em elevações mais aplainadas. Nestas áreas, os solos se apresentam bem desenvolvidos e de textura argilosa. As regiões planas apresentam grande parte de solos transportados (Castañeda 1993).

O mapa da figura 1 apresenta os pontos de amostragem dos solos selecionados de acordo com as litologias de cada região. As coletas de amostras de solos correspondem às regiões denominadas de Manso (Man), Calais (Cal), Benedito (Ben), Belchior (Br), Custódio (Cd) e Mainard (Md). A tabela 1 apresenta as coordenadas geográficas dos pontos amostrados. Em cada ponto foram coletadas amostras nas profundidades de $0-10 \mathrm{~cm}, 10-20 \mathrm{~cm}, 20-30 \mathrm{~cm}, 30-40$ $\mathrm{cm}, 40-50 \mathrm{~cm}$ e $50-60 \mathrm{~cm}$.

O material coletado foi acondicionado em sacos plásticos devidamente etiquetados. Até a realização das análises de laboratório, as amostras de solo foram armazenadas em uma câmara úmida a fim de se conservarem as suas características originais. 


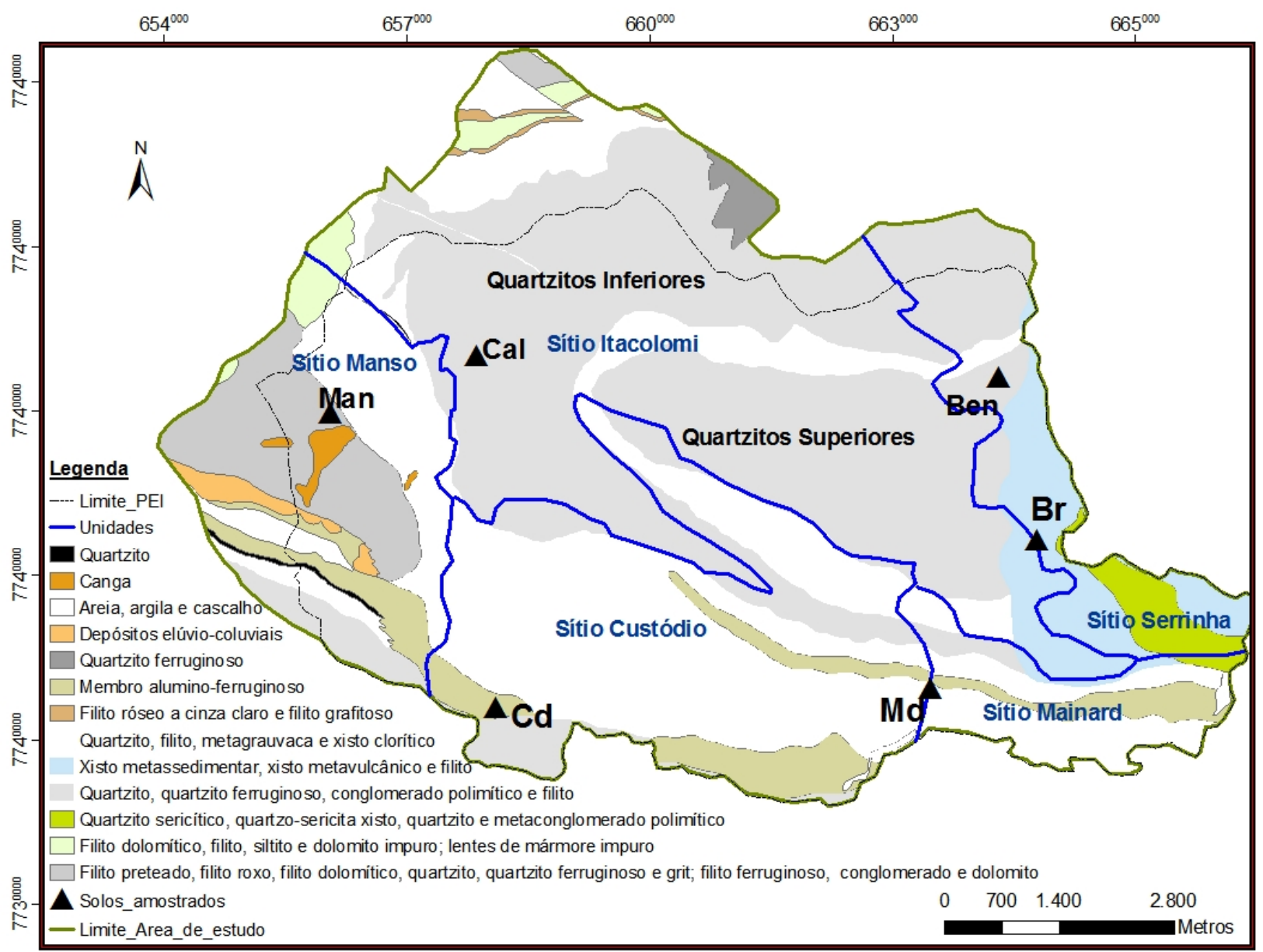

Figura 1: Localização dos solos amostrados (adaptado de Lobato et al. 2004)

Geologicamente, a área de estudo está inserida na porção sudeste do Quadrilátero Ferrífero (QF), uma das áreas clássicas do Pré-Cambriano, situada no extremo sul do Cráton de São Francisco.

A estratigrafia na região do parque é representada por rochas do Supergrupo Rio das Velhas, do Supergrupo Minas (Grupos Piracicaba e Sabará) e pelas rochas metassedimentares clásticas do Grupo Itacolomi (Glöeckner 1981). Os quartzitos do Grupo Itacolomi foram divididos por Ferreira \& Lazarin (1993) em dois subgrupos: inferior e superior, que representam, respectivamente, os quartzitos Morro do Cachorro (QMC) e os quartzitos Pico do Itacolomi (QPI), separados por xistos do Grupo Sabará. Encontram-se, também, na área, rochas intrusivas básicas e depósitos recentes de laterita (Glöeckner 1981). O Supergrupo Rio das Velhas, na área do parque, se divide nos Grupos Maquiné e Nova Lima e apresenta sequências vulcano-sedimentares arqueanas do tipo greenstone belt.

O Grupo Sabará, pertencente ao SG Minas, é constituído, basicamente, por xistos, sendo que ao topo dessa formação é comum encontrar afloramentos de filito e quartzito ferruginoso. O Grupo Piracicaba, na área, é composto principalmente por filito grafitoso. Os quartzitos inferiores (QMC) do Grupo Itacolomi apresentam litofácies como metaconglomerados, metarenitos e quartzitos ferruginosos. Os quartzitos superiores (QPI) ocorrem em contato tectônico (falhas de empurrão) com xistos do Grupo Sabará no topo da sequência Itacolomi e apresentam um conteúdo sericítico. Os depósitos recentes, presentes na área do parque, são representados por coluviões, aluviões e níveis lateríticos superficiais de canga (Ferreira \& Lazarin 1993).

\subsection{Determinação da geoquímica dos solos}

As concentrações dos elementos foram determinadas via Espectrometria de Emissão Atômica com fonte Plasma Indutivamente Acoplado (ICP-OES) (APHA 1992), no Laboratório de Geoquímica Ambiental (LGqa) do Departamento de Geologia da Universidade Federal de Ouro Preto (DEGEO/UFOP).

Para a análise das amostras de solos foi utilizado o método de digestão total (Moutte 1990). Num recipiente de savilex de $30 \mathrm{ml}$, foram adicionados 0,25g de solo pulverizado junto com $1 \mathrm{ml}$ de $\mathrm{HNO}_{3}$ (10 
mol/L). Logo após, o savilex aberto foi aquecido a $110^{\circ} \mathrm{C}$, em uma chapa, até secagem incipiente. Posteriormente, foram adicionados $3 \mathrm{ml}$ de $\mathrm{HF}$ concentrado, e o savilex foi aquecido novamente até a secagem. Foram acrescentados mais $3 \mathrm{ml}$ de HF concentrado e os savilex foram fechados e aquecidos a $140^{\circ} \mathrm{C}$ por 16 horas. Após este aquecimento, os recipientes foram resfriados e novamente aquecidos à temperatura de $140{ }^{\circ} \mathrm{C}$ até a secagem. Após o resfriamento foram adicionados $3 \mathrm{ml}$ de $\mathrm{HNO} 3$ $(10 \mathrm{~mol} / \mathrm{L})$, com posterior aquecimento a $110^{\circ} \mathrm{C}$ até a secagem. Foram acrescentados $3 \mathrm{ml}$ de $\mathrm{HCl}$ (10 $\mathrm{mol} / \mathrm{L})$, com aquecimento a $110^{\circ} \mathrm{C}$ até a secagem. Finalmente, $25 \mathrm{ml}$ de $\mathrm{HCl}(2 \mathrm{~mol} / \mathrm{L})$ foram adicionados, e os recipientes fechados para aquecimento a $110^{\circ} \mathrm{C}$ por duas horas, com o objetivo de redissolver os sais. Após o resfriamento, o material foi pesado (savilex + amostra) e a solução resultante analisada.

\subsection{Análise mineralógica e granulométrica}

A composição mineralógica dos solos foi determinada por meio de difração de raios-X (Brindley \& Brown 1984) utilizando o aparelho Rigaku, modelo D/Max-2B, com radiação CuK, corrente de $15 \mathrm{~mA}$ e voltagem de $40 \mathrm{KV}$. A análise química total dos elementos maiores (óxidos) foi realizada por meio de fluorescência de raios-X, neste mesmo equipamento, no LGqa (DEGEO/UFOP).

Também foi realizada a caracterização granulométrica dos solos, determinada por peneiramento e sedimentação (NBR-7181/ABNT).

Uma quantidade representativa de material foi selecionada e sua umidade determinada. Posteriormente, este material foi passado na peneira \#10 (2,0 mm) e separado em três quantidades: 50g para a determinação do peso específico real das partículas, $70 \mathrm{~g}$ para a sedimentação e $50 \mathrm{~g}$ para o peneiramento fino. Para o peneiramento grosso (material retido na peneira \#10), o material foi lavado na peneira \#10 (2,0 $\mathrm{mm}$ ) e em seguida colocado na estufa. $\mathrm{O}$ material seco foi peneirado mecanicamente até a peneira \#10, e a fração retida em cada peneira foi pesada. Para o peneiramento fino (material que passa na peneira \#10), o material foi lavado na peneira \#200 $(0,075 \mathrm{~mm})$ e colocado na estufa. O material seco foi passado nas peneiras de aberturas menores que a \#10 e a fração retida em cada peneira foi pesada. Na sedimentação, o material foi colocado em "banho" (6 a 24 horas) sem defloculante.

A mistura foi agitada no dispersor elétrico por 15 minutos, sendo transferida posteriormente para a proveta graduada, sendo esta completada com água destilada até $1.000 \mathrm{ml}$. Por fim, foram realizadas leituras com densímetro nos tempos de $15 \mathrm{seg}, 30 \mathrm{seg}$; $1 \mathrm{~min}, 2 \mathrm{~min}, 4 \mathrm{~min}, 8 \mathrm{~min}, 15 \mathrm{~min}, 30 \mathrm{~min}$; $1 \mathrm{~h}$, 2h, 4h, 8h, 24h.

Também no Departamento de Geologia (DEGEO) foi determinada a concentração de matéria orgânica no solo por perda ao fogo (ou método de calcinação).
Cerca de $1 \mathrm{~g}$ de cada amostra previamente seca foi levada a mufla e aquecida a $750^{\circ} \mathrm{C}$. Feito isso, a amostra foi pesada e a diferença entre os pesos inicial e final correspondeu ao teor de matéria orgânica (Davies 1974).

\section{Resultados e Discussão}

Os resultados obtidos foram comparados com os valores-padrões de solos definidos pela norma CETESB 195/05. Tais valores representam a concentração de determinada substância no solo que o define como não contaminado.

De acordo com CETESB (2005), os valores de referência para As, Ba, Cd, Co, Cr, Ni, Pb e Zn são de $3,5 \mathrm{mg} / \mathrm{kg}, 75 \mathrm{mg} / \mathrm{kg}, 0,5 \mathrm{mg} / \mathrm{kg}, 13 \mathrm{mg} / \mathrm{kg}, 40 \mathrm{mg} / \mathrm{kg}$, $13 \mathrm{mg} / \mathrm{kg}, 17 \mathrm{mg} / \mathrm{kg}$ e de $60 \mathrm{mg} / \mathrm{kg}$, respectivamente. É interessante destacar que a CETESB (2005) não registra os valores de referência para todos os elementos analisados. Baseado nestes valores pode-se observar que a maior parte dos solos apresenta valores superiores à norma (tabela 2), e como a área de estudo é uma Unidade de Conservação, com menor possibilidade de interferência antrópica, pode-se dizer que a concentração elevada de tais elementos é uma resposta química natural das características geológicas de cada área.

Nos solos da sub-bacia do rio Manso foram encontradas elevadas concentrações dos elementos Fe, $\mathrm{Mn}$, As e Al, refletindo a geologia local composta por quartzitos ferruginosos, faixas de canga, xistos e filitos. Também foram encontradas altas concentrações de Mn e Fe na sub-bacia do rio Calais, e de Al na sub-bacia do rio Benedito.

Estas elevadas concentrações têm como principais fontes o quartzito ferruginoso (no Grupo Sabará), os xistos e filitos do grupo Piracicaba. As concentrações anômalas de alumínio, verificadas na sub-bacia do rio Benedito, podem ser atribuídas aos quartzitos sericíticos.

Com base na resolução CETESB (2005), pode-se concluir que os solos do Manso apresentam valores anômalos de As, Pb, Zn, Cd e Cr. Anomalias de Ba e Co foram verificadas em todas as amostras de solo, e anomalias de $\mathrm{Cr}$ e Cd somente não foram encontrados nos solos do Calais. Os solos do Custódio e Belchior apresentaram concentrações anômalas de $\mathrm{Pb}$ e $\mathrm{Zn}$. Nos solos do Benedito, Mainard e Calais foram verificadas concentrações anômalas de Zn.

Na tabela 3 é apresentada a composição mineralógica dos solos amostrados. A área é constituída principalmente por minerais de quartzo, muscovita, caulinita, goethita e gibsita. Como se pode observar na tabela 2, os elementos químicos $\mathrm{Fe}$ e $\mathrm{Al}$ são os mais abundantes nas amostras analisadas, confirmando os resultados obtidos na difração de raios-X referente aos minerais quartzo, caulinita e gibsita. Os óxidos de titânio, manganês, fósforo, magnésio e cálcio são encontrados em pequenas quantidades, geralmente não ultrapassando $1 \%$ na maioria das amostras (tabela 3). 
Tabela 2: Teor de elementos selecionados em amostras de solo

\begin{tabular}{|c|c|c|c|c|c|c|c|c|c|c|c|c|c|c|c|c|c|c|c|c|}
\hline $\begin{array}{l}\text { Pontos } \\
\text { amost. }\end{array}$ & $\begin{array}{l}\text { Classif. } \\
\text { solos }\end{array}$ & $\begin{array}{c}\text { Prof. } \\
\text { Coleta }\end{array}$ & $\begin{array}{c}\mathrm{Al} \\
\mathrm{mg} / \mathrm{kg}\end{array}$ & Fé $\mathrm{mg} / \mathrm{kg}$ & $\begin{array}{c}\mathrm{Mg} \\
\mathrm{mg} / \mathrm{kg}\end{array}$ & $\begin{array}{c}\text { Mn } \\
\mathrm{mg} / \mathrm{kg}\end{array}$ & $\begin{array}{c}\text { As } \\
\mathrm{mg} / \mathrm{kg}\end{array}$ & $\begin{array}{c}\mathbf{C r} \\
\mathrm{mg} / \mathrm{kg}\end{array}$ & $\begin{array}{c}\mathbf{C d} \\
\mathrm{mg} / \mathrm{kg}\end{array}$ & $\begin{array}{c}\mathbf{P b} \\
\mathrm{mg} / \mathrm{kg}\end{array}$ & $\begin{array}{c}\mathrm{Zn} \\
\mathrm{mg} / \mathrm{kg}\end{array}$ & $\begin{array}{c}\text { Co } \\
\mathrm{mg} / \mathrm{kg}\end{array}$ & $\begin{array}{c}\mathrm{Cu} \\
\mathrm{mg} / \mathrm{kg}\end{array}$ & $\begin{array}{c}\mathbf{B a} \\
\mathrm{mg} / \mathrm{kg}\end{array}$ & $\begin{array}{c}\mathrm{Ca} \\
\mathrm{mg} / \mathrm{kg}\end{array}$ & $\begin{array}{c}\mathbf{K} \\
\mathrm{mg} / \mathrm{kg}\end{array}$ & $\begin{array}{c}\mathrm{Na} \\
\mathrm{mg} / \mathrm{kg}\end{array}$ & $\begin{array}{c}\mathbf{N i} \\
\mathrm{mg} / \mathrm{kg}\end{array}$ & $\begin{array}{c}\mathrm{Sr} \\
\mathrm{mg} / \mathrm{kg}\end{array}$ & $\begin{array}{c}\mathbf{Z r} \\
\mathrm{mg} / \mathrm{kg}\end{array}$ \\
\hline CETESB $^{(1)}$ & & & & & & & 3,5 & 40 & 0,5 & 17 & 60 & 13 & & & & & & & & \\
\hline \multirow{7}{*}{ Cal } & \multirow{7}{*}{$\begin{array}{c}\text { Neossolo } \\
\text { Litólico }\end{array}$} & $0-10$ & 38308 & 6186 & 612 & 30,85 & $<\mathrm{LQ}$ & $<\mathrm{LQ}$ & $<\mathrm{LQ}$ & $<\mathrm{LQ}$ & $<\mathrm{LQ}$ & 25,0 & $<\mathrm{LQ}$ & 697 & $<\mathrm{LQ}$ & 14575 & $<\mathrm{LQ}$ & 9,35 & 32,94 & 114,4 \\
\hline & & $10-20$ & 40024 & 6775 & 624 & 18,66 & $<\mathrm{LQ}$ & $<\mathrm{LQ}$ & $<\mathrm{LQ}$ & $<\mathrm{LQ}$ & $<\mathrm{LQ}$ & 29,4 & $<\mathrm{LQ}$ & 699 & 42,38 & 14683 & $<\mathrm{LQ}$ & 9,15 & 32,69 & 125,9 \\
\hline & & $20-30$ & 39988 & 6542 & 652 & 12,51 & $<\mathrm{LQ}$ & $<\mathrm{LQ}$ & $<\mathrm{LQ}$ & $<\mathrm{LQ}$ & $<\mathrm{LQ}$ & 26,6 & $<\mathrm{LQ}$ & 726 & 16,15 & 15691 & $<\mathrm{LQ}$ & 9,94 & 35,08 & 118,2 \\
\hline & & $30-40$ & 52570 & 13991 & 734 & 17,63 & $<\mathrm{LQ}$ & $<\mathrm{LQ}$ & $<\mathrm{LQ}$ & $<\mathrm{LQ}$ & 82 & 24,2 & $<\mathrm{LQ}$ & 879 & 49,04 & 19207 & $<\mathrm{LQ}$ & 9,38 & 41,33 & 117,4 \\
\hline & & $40-50$ & 55662 & 11871 & 790 & 16,10 & $<\mathrm{LQ}$ & $<\mathrm{LQ}$ & $<\mathrm{LQ}$ & $<\mathrm{LQ}$ & $<\mathrm{LQ}$ & 21,9 & $<\mathrm{LQ}$ & 932 & 32,92 & 21002 & $<\mathrm{LQ}$ & 10,44 & 43,31 & 135,6 \\
\hline & & $50-60$ & 57306 & 9358 & 831 & 11,99 & $<\mathrm{LQ}$ & $<\mathrm{LQ}$ & $<\mathrm{LQ}$ & $<\mathrm{LQ}$ & 571 & 21,3 & $<\mathrm{LQ}$ & 989 & 221,5 & 21803 & $<\mathrm{LQ}$ & 11,47 & 49,83 & 121,5 \\
\hline & & $0-10$ & 53827 & 44860 & 593 & 138,20 & $<\mathrm{LQ}$ & 96,4 & 4,7 & $<\mathrm{LQ}$ & $<\mathrm{LQ}$ & 27,9 & 10,7 & 295 & $<\mathrm{LQ}$ & 8586 & 1753 & 20,82 & 44,3 & 75,9 \\
\hline \multirow[t]{6}{*}{ Ben } & \multirow{6}{*}{$\begin{array}{l}\text { Neossolo } \\
\text { Litólico }\end{array}$} & $10-20$ & 67528 & 52361 & 694 & 158,90 & $<\mathrm{LQ}$ & 113,1 & 5,3 & $<\mathrm{LQ}$ & $<\mathrm{LQ}$ & 38,8 & 14,1 & 356 & $<\mathrm{LQ}$ & 10255 & 2120 & 24,49 & 53,5 & 89,3 \\
\hline & & $20-30$ & 64872 & 52049 & 711 & 181,00 & $<\mathrm{LQ}$ & 103,5 & 5,3 & $<\mathrm{LQ}$ & $<\mathrm{LQ}$ & 33,1 & 16,2 & 349 & $<\mathrm{LQ}$ & 9709 & 2030 & 24,49 & 51,6 & 70,9 \\
\hline & & $30-40$ & 65703 & 48109 & 788 & 142,10 & $<\mathrm{LQ}$ & 107,4 & 4,9 & $<\mathrm{LQ}$ & $<\mathrm{LQ}$ & 39,2 & 13,7 & 362 & $<\mathrm{LQ}$ & 10912 & 2121 & 23,59 & 51,1 & 89,8 \\
\hline & & $40-50$ & 60807 & 42155 & 673 & 162,30 & $<\mathrm{LQ}$ & 96,0 & 3,9 & $<\mathrm{LQ}$ & $<\mathrm{LQ}$ & 24,5 & 11,2 & 354 & $<\mathrm{LQ}$ & 10021 & 2001 & 21,20 & 55,6 & 83,4 \\
\hline & & $50-60$ & 77208 & 41079 & 793 & 117,60 & $<\mathrm{LQ}$ & 120,3 & 3,3 & $<\mathrm{LQ}$ & $<\mathrm{LQ}$ & 24,1 & 12,9 & 489 & $<\mathrm{LQ}$ & 15063 & 2712 & 25,18 & 68,9 & 82,2 \\
\hline & & $0-10$ & 96704 & 241975 & 792 & 1378 & 184,1 & 380,0 & 43,3 & 53 & 352,6 & 42,1 & 12,9 & 385 & 194,0 & 9625 & 1460 & 94,50 & 24,9 & 200,1 \\
\hline \multirow[t]{6}{*}{$\underline{\text { Man }}$} & \multirow[t]{6}{*}{ Latossolo } & $10-20$ & 93082 & 263338 & 694 & 2234 & 238,7 & 409,0 & 48,2 & 94 & 339,7 & 49,4 & 7,8 & 403 & 175,8 & 8902 & 1284 & 120,5 & 26,2 & 184,9 \\
\hline & & $20-30$ & 107870 & 216596 & 876 & 2337 & 200,6 & 388,6 & 37,5 & 103 & 384,4 & 46,0 & 27,0 & 421 & 194,7 & 11785 & 1516 & 106,9 & 31,5 & 212,0 \\
\hline & & $30-40$ & 121260 & 192081 & 1062 & 1260 & 146,4 & 363,1 & 31,8 & 48 & 291,8 & 34,0 & 9,0 & 453 & 150,9 & 15135 & 1910 & 96,90 & 34,1 & 213,9 \\
\hline & & $40-50$ & 123795 & 114487 & 1080 & 582 & 78,5 & 331,6 & 18,8 & 46 & 472,1 & 32,6 & 39,0 & 391 & 873,0 & 13638 & 2257 & 150,1 & 40,8 & 201,9 \\
\hline & & $50-60$ & 140064 & 86540 & 1383 & 349 & 46,5 & 322,1 & 9,5 & $<\mathrm{LQ}$ & 88,3 & 18,1 & 55.0 & 514 & $<\mathrm{LQ}$ & 19821 & 3658 & 84,80 & 48,3 & 202,7 \\
\hline & & $0-10$ & 92407 & 88942 & 4411 & 396 & $<\mathrm{LQ}$ & 279,9 & 11,4 & 46 & $<\mathrm{LQ}$ & 27,0 & 22,2 & 331 & $<\mathrm{LQ}$ & 11364 & 810 & 57,80 & 19,9 & 196,1 \\
\hline \multirow[t]{6}{*}{$\underline{\text { Cd }}$} & \multirow[t]{6}{*}{ Latossolo } & $10-20$ & 95173 & 87888 & 4461 & 342 & $<\mathrm{LQ}$ & 300,6 & 11,0 & 42 & 93,9 & 27,3 & 21,7 & 333 & $<\mathrm{LQ}$ & 11866 & 841 & 58,30 & 22,3 & 180,3 \\
\hline & & $20-30$ & 104475 & 101243 & 4185 & 431 & $<\mathrm{LQ}$ & 283,4 & 13,1 & 49 & $<\mathrm{LQ}$ & 31,7 & 27,0 & 319 & $<\mathrm{LQ}$ & 12329 & 816 & 60,90 & 18,5 & 181,4 \\
\hline & & $30-40$ & 99680 & 96205 & 4102 & 387 & $<\mathrm{LQ}$ & 287,6 & 13,1 & 58 & 75,1 & 34,1 & 23,0 & 315 & $<\mathrm{LQ}$ & 11922 & 813 & 57,70 & 18,9 & 181,2 \\
\hline & & $40-50$ & 95290 & 87444 & 4656 & 340 & $<\mathrm{LQ}$ & 294,2 & 11,0 & 39 & 79,6 & 34,6 & 22,6 & 347 & $<\mathrm{LQ}$ & 12159 & 910 & 60,30 & 22,9 & 180,9 \\
\hline & & $50-60$ & 90676 & 77210 & 4694 & 275 & $<\mathrm{LQ}$ & 309,8 & 9,5 & 29 & $<\mathrm{LQ}$ & 32,9 & 17,7 & 361 & $<\mathrm{LQ}$ & 12534 & 922 & 59,20 & 25,3 & 195,4 \\
\hline & & $0-10$ & 141576 & 59883 & 1302 & 261 & $<\mathrm{LQ}$ & 193 & 4,4 & $<\mathrm{LQ}$ & $<\mathrm{LQ}$ & 22,2 & 33,5 & 1140 & 65,7 & 30266 & 6438 & 66,60 & 124,7 & 101,6 \\
\hline \multirow{6}{*}{$\underline{\mathrm{Br}}$} & \multirow[t]{6}{*}{ Latossolo } & $10-20$ & 161283 & 58836 & 1485 & 141 & $<\mathrm{LQ}$ & 220,8 & 6,5 & 57 & 239,2 & 16,1 & $<\mathrm{LQ}$ & 1300 & 492 & 34821 & 8590 & 53,10 & 163,7 & 118,4 \\
\hline & & $20-30$ & 343221 & 159588 & 3501 & 1019 & $<\mathrm{LQ}$ & 465,9 & 13,9 & 26 & 29,1 & 68,9 & $<\mathrm{LQ}$ & 2791 & 85,5 & 73182 & 14590 & 173,5 & 299,3 & 269,3 \\
\hline & & $30-40$ & 141346 & 65429 & 1387 & 322 & $<\mathrm{LQ}$ & 201,3 & 5,2 & $<\mathrm{LQ}$ & $<\mathrm{LQ}$ & 23,1 & 34,2 & 1089 & 16,3 & 28829 & 5570 & 70,40 & 117,5 & 128,4 \\
\hline & & $40-50$ & 135505 & 60030 & 1424 & 492 & $<\mathrm{LQ}$ & 188,0 & 5,1 & $<\mathrm{LQ}$ & $<\mathrm{LQ}$ & 26,2 & 34,4 & 1042 & $<\mathrm{LQ}$ & 26983 & 4900 & 73,00 & 103,0 & 113,8 \\
\hline & & $50-60$ & 135773 & 62284 & 1424 & 340 & $<\mathrm{LQ}$ & 198,0 & 5,0 & $<\mathrm{LQ}$ & $<\mathrm{LQ}$ & 25,4 & 34,0 & 1090 & $<\mathrm{LQ}$ & 28260 & 5022 & 72,50 & 114,7 & 126,1 \\
\hline & & $0-10$ & 67834 & 63270 & 2177 & 1201 & $<\mathrm{LQ}$ & 102,4 & 7,9 & $<\mathrm{LQ}$ & 94,6 & 29,2 & 14,7 & 563 & 529 & 18012 & 1457 & 46,41 & 45,1 & 127,8 \\
\hline \multirow[t]{5}{*}{$\underline{\text { Md }}$} & \multirow[t]{5}{*}{ Latossolo } & $10-20$ & 67461 & 66430 & 2270 & 1149 & $<\mathrm{LQ}$ & 104,5 & 8,4 & $<\mathrm{LQ}$ & 74,8 & 26,6 & 14,4 & 541 & 615 & 16889 & 1563 & 47,28 & 45,6 & 133,7 \\
\hline & & $20-30$ & 65647 & 66630 & 2359 & 951 & $<\mathrm{LQ}$ & 122,3 & 9,5 & $<\mathrm{LQ}$ & 4125 & 29,0 & $<\mathrm{LQ}$ & 535 & 1363 & 15774 & 1696 & 52,70 & 48,0 & 153,3 \\
\hline & & $30-40$ & 48269 & 43011 & 1987 & 692 & $<\mathrm{LQ}$ & 80,8 & 4,8 & $<\mathrm{LQ}$ & 130,5 & 28,5 & 13,3 & 388 & 734 & 10796 & 1406 & 39,08 & 36,3 & 95,1 \\
\hline & & $40-50$ & 41340 & 35572 & 1987 & 532 & $<\mathrm{LQ}$ & 71,7 & 3,6 & $<\mathrm{LQ}$ & 133,5 & 29,7 & 12,3 & 350 & 787 & 9143 & 1400 & 33,46 & 33,7 & 112,3 \\
\hline & & $50-60$ & 48355 & 39158 & 2098 & 600 & $<\mathrm{LQ}$ & 76,4 & 4,1 & $<\mathrm{LQ}$ & 61,7 & 25,8 & 13,8 & 413 & 707 & 11314 & 1528 & 35,38 & 36,1 & 127,6 \\
\hline $\mathbf{L} \mathbf{Q}^{(1)}$ & & & 13,7 & 6,7 & 0,17 & 0,26 & 5,0 & 0,9 & 0,8 & 6,4 & 0,4 & 15 & 0,5 & 0,06 & 4,6 & 52,7 & 3,2 & 2,5 & 0,03 & 0,44 \\
\hline
\end{tabular}

(1) LQ - Limite de quantificação.

(2) Valores da Norma CETESB 195/05 (CETESB 2005). 
Tabela 3: Composição mineralógica dos solos

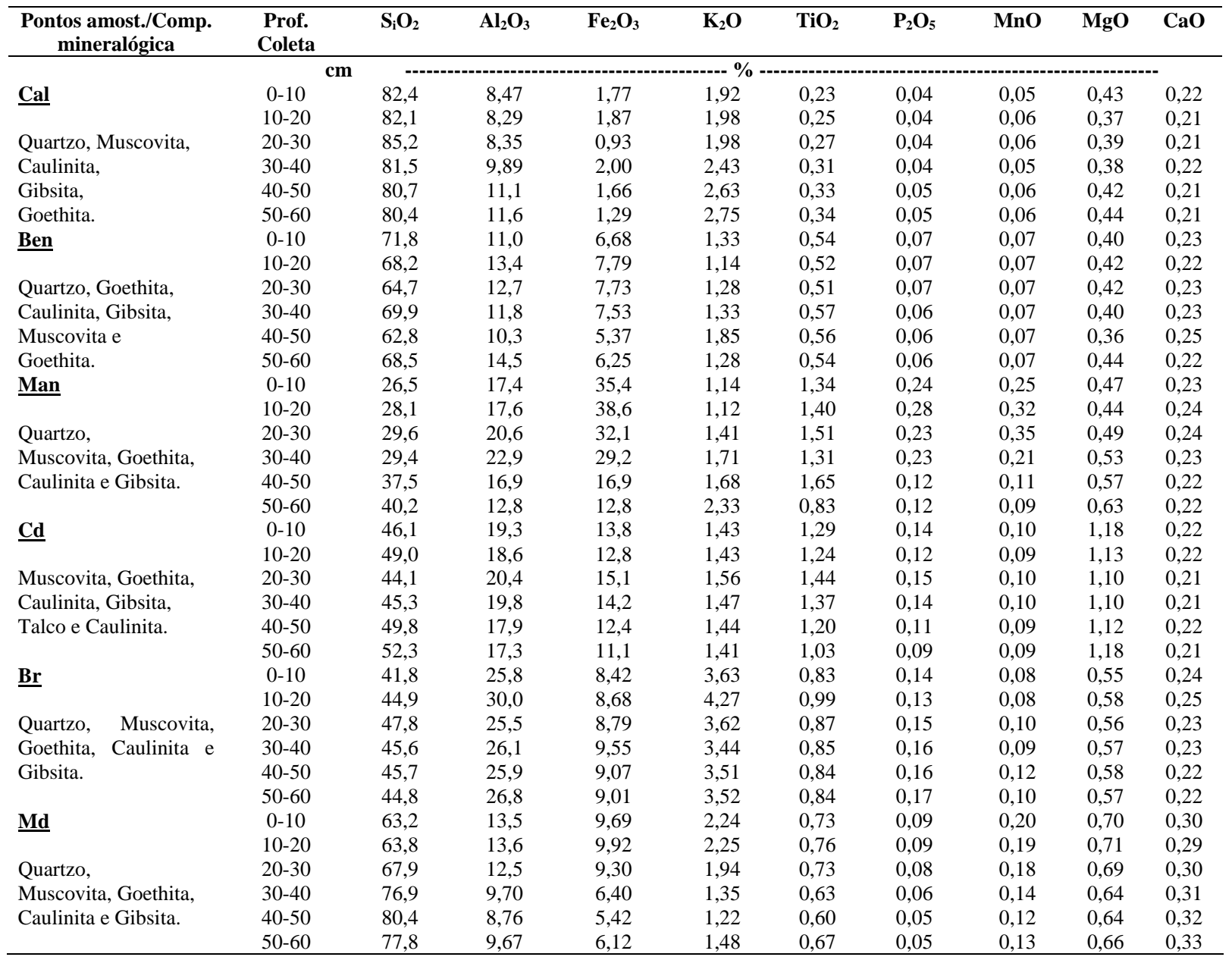

Vale ressaltar que o uso dos valores geoquímicos estabelecidos pela norma CETESB 195/05, como referência para as diversas regiões do Brasil, pode levar a avaliações inadequadas, já que a geoquímica dos solos é litodependente e as condições pedogeomorfológicas bem distintas em cada região.

Na tabela 4 é apresentada a composição granulométrica, a matéria orgânica e o pH dos solos amostrados. Na região do Manso foram encontrados solos siltosos mais desenvolvidos, profundos e mal selecionados. As maiores proporções de matéria orgânica foram encontradas neste solo, com concentrações entre 11,5\% e 16\%.

Os solos do Benedito apresentam certo equilíbrio entre as concentrações de pedregulho, silte, argila e areia fina, média e grossa, e teores de matéria orgânica entre 7,6 e 18,4\%.

As frações de areias finas e médias predominaram nos solos da região do Calais. Além disso, são solos bem selecionados e com baixas concentrações de matéria orgânica. As classes texturais de areia fina e silte representam as maiores frações nas amostras do Mainard.
As camadas dos solos do Belchior apresentaram maiores concentrações de silte e areia fina. As concentrações de matéria orgânica ficaram entre 9,2 e $12 \%$. Já os solos amostrados na região do Custódio apresentaram maiores concentrações de silte e areia fina. As concentrações de matéria orgânica ficaram entre 10,6 e 14,5\%.

Os solos são extremamente ricos em matéria orgânica. A elevada altitude da área faz com que as temperaturas sejam muito baixas, e nestas condições, a decomposição bioquímica da matéria orgânica é retardada.

Os baixos teores de argila apresentados se devem ao experimento realizado sem o uso de defloculante. Ou seja, a argila fica agregada e é contabilizada nas frações silte e areia muito fina. Além disso, a própria pedogênese, temperatura e tectônica da área, com rochas quartzíticas, falhas e fraturas, propiciam a formação de solo arenoso.

O elevado teor de matéria orgânica modifica o sistema, que associado aos baixos valores de $\mathrm{pH}$ e provavelmente baixos valores de Eh, propicia a solução de diversos elementos, sobretudo de metais, 
como $\mathrm{Cr}$, Ni, Fe e $\mathrm{Mn}$, que têm suas concentrações reduzidas.

Nos solos com valores de Eh mais elevados, em geral com menor porcentagem de matéria orgânica, o ambiente oxidante faz com que estes elementos fiquem no solo, sempre havendo entre eles uma correlação. Porém é importante ressaltar que esta correlação existe em função do ambiente, das características químicas e físicas do sistema, e não da presença de $\mathrm{Fe}$ ou $\mathrm{Mn}$, que são elementos abundantes na região do Quadrilátero Ferrífero.

\section{Conclusões}

Os teores extraídos para os elementos $\mathrm{Al}, \mathrm{Fe}, \mathrm{Mg}$, $\mathrm{Mn}, \mathrm{As}, \mathrm{Cr}, \mathrm{Pb}, \mathrm{Zn}$ e Co nos solos das regiões do Calais, Benedito, Manso, Custódio, Belchior e Mainard são relativamente altos e influenciados pela litologia local.

Pelo fato de a área de estudo ser de preservação permanente, com possibilidade de influência antrópica pouco significativa, e mesmo assim apresentar valores bem superiores aos estabelecidos pela resolução CETESB (2005), verifica-se a necessidade de que os valores-padrões de qualidade do solo sejam estabelecidos com base nas características específicas de cada local.

Dessa forma, os resultados encontrados neste estudo podem ser utilizados para a avaliação preliminar de áreas com suspeita de contaminação, cujos solos possuam características geológicas semelhantes às das regiões estudadas. A distribuição dos elementos nos solos está intimamente associada ao material de origem e aos fatores pedogenéticos. Tal fato pode ser observado para os solos do Parque Estadual do Itacolomi, pois, em geral, os teores encontrados de $\mathrm{Fe}, \mathrm{Mn}$, As e $\mathrm{Al}$ expressam a condição de relativa riqueza desses elementos nos materiais parentais, constituídos sobretudo por quartzitos ferruginosos, cangas, xistos, filitos e quartzitos sericíticos.

Tabela 4: Composição granulométrica, matéria orgânica e pH dos solos

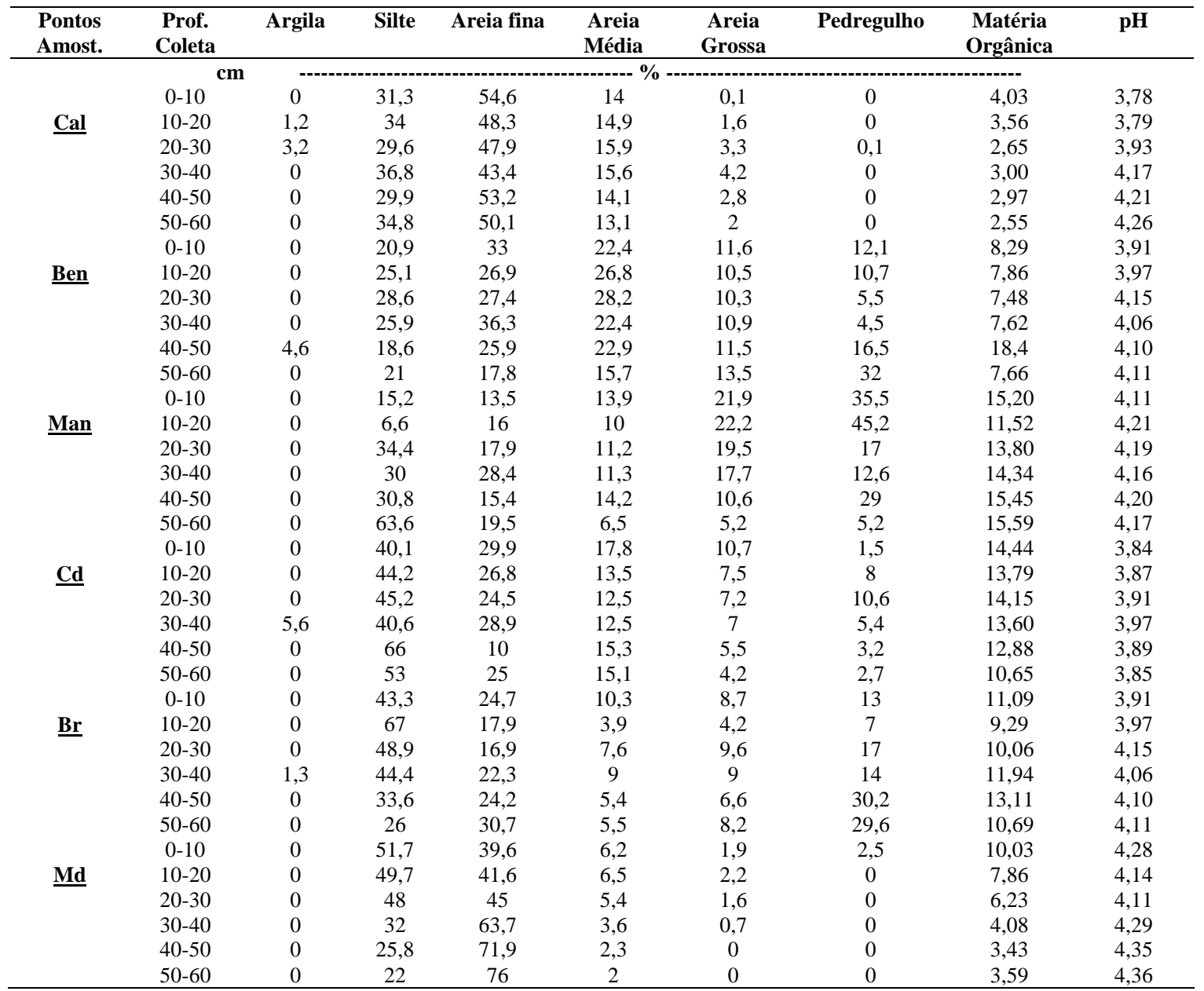




\section{Referências}

Alloway B. J. 1993. Heavy Metals in Soils. John Wiley \& Sons Inc., New York. 553p.

APHA. 1992. Standard Methods for the Examination of Water and Wastewater. $18^{\text {th }}$ edition. American Public Health association (APHA). American Water Works Association (AWWA) and Water Pollution Control Federation (WPCF), Washington, DC.

Barona A., Romero F. 1996. Distribution of metals in soils and relationships among fractions by principal component analysis. Soil Technology, 8: 303-319.

Brindley G. W., Brown G. 1984. Cristal structures of clays minerals and their x-Ray indentification. London. Ed. Mineralogical Society, 495p.

Burak D. L. 2008. Geoquímica e distribuição de metais pesados em solos na região de Unaí, Paracatu e vazante, MG. Tese de Doutorado. Pós-Graduação em Solos e Nutrição de Plantas, Departamento de Agronomia, Universidade Federal de Viçosa, 189p.

Castañeda C. 1993. Caracterização Geológica e Geomorfológica do Parque Estadual de Itacolomi. Projeto Itacolomi. Convênio IEF/UFOP/BIRD, 36 p. (Relatório de Técnico).

CETESB - Companhia de Tecnologia de Saneamento Ambiental. 2005. Decisão de diretoria $N^{\circ}$ 195-2005 da Companhia de Tecnologia de Saneamento Ambiental de São Paulo. São Paulo. 4p.

CONAMA - Conselho Nacional do Meio Ambiente. 2009. Resolução $\mathrm{n}^{\circ} 420$ do Conselho Nacional do Meio Ambiente, de 28 de dezembro de 2009 - Dispõe sobre critérios e valores orientadores de qualidade do solo quanto à presença de substâncias químicas e estabelece diretrizes para o gerenciamento ambiental de áreas contaminadas por essas substâncias em decorrência de atividades antrópicas. Ministério do Meio Ambiente. Brasil, 16 p.

Davies B. E. 1990. Lead. In: Alloway B.J. (ed). Heavy metals in soils. New York, John Wiley, p.177-193.

Davies B. E. 1974. Loss-on-ignition as an Estimate of Soil Organic Matter. Soil Sci. Soc. Am. Proc., 38:347-353.

Fadigas F., Sobrinho, N. M. B. A., Mazur, N., Anjos, L. H. C. A., Freixo, A.A. 2006. Proposição de valores de referência para a concentração natural de metais pesados em solos brasileiros. Revista Brasileira de Engenharia Agrícola e Ambiental, 10: 699705.

Ferreira A. F., Lazarin, H. A. 1993. Caracterização litoestrutural e geomorfológica da região do Pico do Itacolomi, Ouro Preto, MG. Monografia de Graduação. Departamento de Geologia, Universidade Federal de Ouro Preto, 54p.

Glöeckner K. J. 1981. Lithostratigraphic. Sedimentologie, Tektonic und Metamoephose der Proterozoischem Itacolomi Serie bei Ouro Preto, Minas Gerais. Brasilien., Ph.D. Thesis, Univ. Clausthal, RFA, 221p.

Gough L. P., Severson R. C., Jackson L. L. 1994. Baseline element concentrations in soils and plants, Bull Island, Cape Romain National Wildlife Refuge, South Carolina, USA. Water Air Soil Poll., 74:1-17.

Hursthouse A. S. 2001. The relevance of speciation in the remediation of soils and sediments contaminated by metallic elements-an overview with specific examples from central Scotland. Journal Environmental Monitoring, 3:49-60.

Klamt E., Reeuwijk L. P. 2000. Evaluation of morphological, physical and chemical characteristics of Ferralsols and related soils. R Bras. Ci. Solo, 24:573-587.

Lobato L.M., Achstschin A.B., Timbó M.A., Baltazar O.F., Silva S. L., Reis L. B., Baars F. J. (2004). Geologia do Quadrilátero Ferrífero: integração e correção cartográfica em SIG. Codemig. Universidade Federal Minas Gerais, Belo Horizonte, CD-room..

Mitchell R. L. 1964. Trace elements in soils. In: Bear F.E. (ed.) Chemistry of the soil. New York, Reinhold, p.320- 368.

Moutte J. 1990. Procedure for multiacid digestion of rocks and minerals Géochimine. École des Mines de Saint Etienne, France. Disponível em: <http:/www.emse.fr/moutte/enplasma/prepar.> Acesso em 9 julho 2009.

ABNT - Associação Brasileira de Normas Técnicas. 1984. NBR 7181: Solo - Análise granulométrica. Rio de Janeiro, 13p.

Oliveira T. S. 1996. Metais pesados como indicadores de materiais de origem de solos. Tese de Doutorado. Pós-Graduação em
Solos e Nutrição de Plantas, Departamento de Agronomia, Universidade Federal de Viçosa, 128p.

Paye H.S. 2008. Valores de referência de qualidade para metais pesados em solos no estado do Espírito Santo. Dissertação de Mestrado. Pós-Graduação em Solos e Nutrição de Plantas, Departamento de Agronomia, Universidade Federal de Viçosa, $64 p$

Souza M. L. P., Andreoli C. V., Amaral M. B. 1996. Levantamento preliminar dos teores de metais pesados em alguns solos do Paraná. Revista Sanare, 5:68-75.

Tiller K. G. 1989. Heavy metals in soil and their environmental significance. Advances in Soil Science, 9:113-142. ${ }^{\mathrm{i}}$

Recebido 14 de fevereiro de 2010 Aceito 28 de novembro de 2011 